\title{
The Effects of Sitting in a Crossed Legs Posture on the Vertebral Angle, Chest Wall Mobility, Pulmonary Function, and Respiratory Muscle Activity: A Preliminary Study
}

\author{
Hee-Eun Ahn, MSc, PT $\cdot$ Tae-Lim Yoon, PhD, $\mathrm{PT}^{1 \dagger}$ \\ Department of Rehabilitation Medicine, Cheongju Samsung Rehabilitation Hospital \\ ${ }^{1}$ Department of Physical Therapy, College of Health and Medical Science, Cheongju University
}

Received: April 3, 2019 / Revised: April 15, 2019 / Accepted: May 31, 2019

(C) 2019 J Korean Soc Phys Med

\section{| Abstract |}

PURPOSE: Sitting with crossed legs may have an effect on maintaining a healthy body posture and proper functioning of the respiratory system. Thus, this study's objective was to identify whether or not sitting with crossed legs affects the vertebral angle, chest wall mobility, the pulmonary function, and the activity of the respiratory muscles.

METHODS: Thirty healthy subjects were recruited for this study (16 males and 14 females). The vertebral angle, chest wall mobility, pulmonary function, and the activity of the respiratory muscle were measured while the subjects sat in the correct posture and these factors were again measured with the subjects seated with their legs crossed. Three-dimensional motion analysis was used to determine the trunk and lumbar vertebral angles. Surface electromyography was employed to measure the sternocleidomastoid, the rectus abdominis, and the external and internal oblique abdominis muscles. A tapeline was utilized to evaluate the subjects' chest wall

†Corresponding Author : Tae-Lim Yoon

free0829@gmail.com, http://orcid.org/0000-0002-1718-2205

This is an Open Access article distributed under the terms of the Creative Commons Attribution Non-Commercial License (http://creativecommons.org/licenses/by-nc/3.0) which permits unrestricted non-commercial use, distribution, and reproduction in any medium, provided the original work is properly cited. mobility. Spirometry was assessed to determine the forced vital capacity and forced expiratory volume in one second. Paired t-tests were then performed $(\mathrm{p}<.05)$.

RESULTS: There were significant differences in the trunk and lumbar flexion angles, the chest wall mobility, the activity of the right external oblique muscle, and the left internal oblique abdominis muscle. However, the difference in pulmonary function did not reach statistical significance.

CONCLUSION: A crossed leg posture caused slight thoracic extension and lumbar flexion, which may lead to a decrease of the chest wall mobility and also to an imbalance of the abdominal muscles. Therefore, sitting with a crossed leg posture should be avoided. Yet a crossed leg posture did not have any clinical effect on the pulmonary function of healthy people. It may be necessary to study the effects of sitting with crossed legs over an extended period of time for patients suffering with impaired respiratory function.

Key Words: Chest mobility, Crossed legs, Muscle activity, Respiration

\section{Introduction}

People are currently spending more time each day in a sitting position and they tend to sit with their legs crossed [1]. A recent study reported three main crossed leg postures: 
one involves sitting with the legs crossed on both sides, the second involves sitting with a leg crossed on the other side of the opposite knee and the thighs are laying atop one another, and the last involves sitting with the leg crossed on the other side of the ankle [2]. In terms of kinematics and kinetic changes, the sitting position with the thighs and legs crossing together has a significant influence on the body.

Sitting with crossed legs results in muscle imbalance, which causes spinal deformity. A leg-crossed posture can lead to problems within the musculoskeletal system by causing the body to deviate from its correct alignment and deforming the trunk's structure [3]. An asymmetrical sitting posture can induce trunk asymmetry by asymmetrically using the abdominal internal oblique (IO) and external oblique (EO) muscles and this can increase the range of motion at the trunk by rotating the pelvis if the range of motion of the hip joint is limited [4]. Also, a crossed leg posture can induce pelvic tilt, leading to scoliosis of the vertebral column [2]. In addition, tilting of the pelvis because of a crossed leg posture may cause asymmetric compression of the buttocks and also scoliosis [5]. Thus, an asymmetric sitting posture may cause permanent spinal deformities, such as kyphosis, scoliosis, and lordosis, and the final result can be chronic lower back pain as well [6].

Changes in the vertebral spine angle can affect the mobility of the chest wall, resulting in changes in the respiratory muscles' activity and function. Changes in the posture can affect the length and stability of the respiratory muscles, resulting in changes in the activity of the respiratory muscles [7]. A previous study claimed that lateral vertebral deformity leads to limitations of the chest wall mobility, resulting in a decreased lung volume and weakening of the respiratory muscles [7]. Another study also reported that a change in posture contributes to altered elasticity of the thoracic chest wall, which affected the respiratory muscles' activity [8]. The changes in the chest wall due to spinal deformity are associated with airway obstruction, inflation, and mechanical shortcomings of the respiratory muscles [8]. When examining the changes of pulmonary function, several studies have commonly investigated the Forced Vital Capacity (FVC), the Forced Expiratory Volume (FEV1), and the activity of the respiratory muscles.

Despite the possibility of inducing changes of the pulmonary function by sitting with crossed legs, there have been no reports on the effects of a crossed leg posture on pulmonary function and the respiratory muscles' pattern of activity. Therefore, we conducted this study to show the effect of sitting with crossed legs on the vertebral angle, the mobility of the chest wall, the pulmonary function, and the activity of the respiratory muscles. This study hypothesized that a crossed leg posture will affect the vertebral angle, the chest wall mobility, the pulmonary function, and respiratory muscle activity.

\section{Methods}

\section{Subjects}

Thirty healthy subjects (height $=167.85 \pm 9.65 \mathrm{~cm}$; weight $=64.57 \pm 15.72 \mathrm{~kg}$; age $=22.88 \pm 1.72$ years; gender $=16$ males and 14 females) were recruited for this study. G power was used to perform calculations on the sample size $(n=25)$ with effect size and statistical power. The effect size was set at approximately .52 and the statistical power (1- $\beta)$ was set at .8. Subjects with movement disorders, pain, a pathological condition of the vertebrae or lower extremities that required treatment during the previous 6 months, and with a past or present history of taking drugs related to respiratory diseases were excluded [2]. All the study's participants provided consent for participating in the study before the experiments [2]. The subjects of this study voluntarily participated in the experiments and signed the experimental agreement. This study was conducted according to the guidelines of the Cheongju University Institutional Review Board (19-A-00000598). 


\section{Equipment}

\section{1) Vertebral Angle measurement}

MyoMotion (MyoMotion research pro, Noraxon Inc., German) uses an inertial measurement unit (IMU) sensor as a wireless motion capture device. The inertial measurement device combines information on angular velocity and acceleration from a geomagnetic sensor to provide data on direction [9]. A small IMU was attached on a body segment, and it tracks the body's 3D angular orientation. By attaching individual IMU sensors on two neighbouring body segments, one can estimate the range of motion of the joint between these two segments. Special fixation straps (for the pelvis) and elastic straps were all used to attach the sensors. Calibration was conducted using the upright position so as to determine the value of the $0 \circ$ angles for the joints being studied. The sampling frequency for the inertial sensors was set at $200 \mathrm{~Hz}$ [10]. Five sensors were attached over the bony landmarks of the external occipital protuberance, the seventh cervical vertebra (C7), the twelfth thoracic vertebra (T12), and the bony areas of the sacrum and femurs [11].

\section{2) Chest wall mobility measurement}

We used a tape measure to measure chest mobility for measuring the degree of change of the chest during breathing. Measurements were made at a position that horizontally crosses the junction between the xiphoid process and the body of the sternum. Chest motility was measured when the subject was in a state of stable respiration during rest and inspiration of as much air as possible [12]. The standardized procedure for making measurements was that the observer should keep the 0 points of the tape fixed on the midline of the body so as to be aligned horizontally with the landmarks, whereas the other end of the tape should be kept mobile to allow tape displacement [13].

\section{3) Pulmonary function tests}

Pulmonary function tests were performed to test the subjects' pulmonary function. The Spirometry kit manufactured by Micro Quark (Cosmed, Italy), was used for the present study [14]. Spirometry is the most frequently used technique to measure pulmonary function, and it is a measure of the amount of respiratory over time [15]. FVC and FEV1 were measured using the Micro Quark Spirometry kit.

For the spirometry measurements, the subject breathed a few times calmly in a single breath, followed by breathing for a maximum of 6 seconds to maintain the FVC and inspiratory vital capacity [14]. The subjects sat in the correct sitting posture with their legs crossed. They exhaled air and then they put in the mouthpiece, internally closed their nasal cavity so that the air did not enter the nasal cavity, and next they drinks the air to their lungs as much as possible and then held it in to swell the chest to their maximum. After three easy, relaxed breaths, the subjects are then asked to aspirate as much air as possible during the subsequent fourth inspiration and then exhale their lung air as quickly as possible. All the pulmonary function parameters were measured as mean values after three measurements.

\section{4) Muscle activity measurement}

An electromyography unit (Telemyo direct transmission system, Noraxon, USA) was used to measure the activity of the respiratory muscles during deep breathing [16]. After skin preparation, disposable $\mathrm{Ag} / \mathrm{Ag}$ CI surface electrodes (15 ' 15 mm; Bioprotech Inc., Wonju, South Korea) were attached to the sternocleidomastoid (SCM), rectus abdominis (RA), EO, and IO muscles. On the SCM, the electrodes were placed at the midpoint between the mastoid process and the clavicle [17], and on the RA, the electrodes were attached about $2 \mathrm{~cm}$ adjacent to the umbilicus. On the EO, the electrodes were positioned $15 \mathrm{~cm}$ above the anterior superior iliac spine, and on the IO, the electrodes were placed at the midpoint between the anterior superior iliac spine and the horizontal umbilicus line [18]. 
Quantification of the EMG values after EMG attachment (maximal voluntary isometric at the postural strength test posture for normalization contraction; MVIC) was performed to measure muscle activity. the standard manual muscle-test positions were used as follows: SCM - supine, elbows flexed and hands beside the head, anterolateral flexion of the neck with the face turned toward the non-tested side against resistance above the temporal region of the head by the second investigator [19]. Measurement of the MVIC value of the RA was taken when the maximum resistance was applied to the subject's shoulder when the subject lifted their body with flexing their knees and the subject in the supine position.

Measurement of the MVIC value of the $\mathrm{EO}$ and $\mathrm{TrA} / \mathrm{IO}$ was taken when the subject rotated the erected upper body to the left and right; the evaluator applied resistance on both shoulders. The measurement was repeated in the opposite direction and both sides were measured [20]. The EMG signal was measured for 5 seconds at a sampling rate of $1500 \mathrm{~Hz}$, but only the middle 3 seconds of data were used in the analysis after stabilization of the posture. Measurements in all the postures were obtained 5 times for each subject [2].

\section{Procedures}

A flat horizontal rotating chair without a backrest, adjustable in height, was used to assess the effect of correct sitting and a crossed leg posture. However, although a rotating chair was used, the subject's left foot was fixed on the floor, and the chair was not rotated [2]. The chair was adjusted to a height so that the hip and knee joints of each subject were flexed to 90 degrees. A familiarization session was conducted before collecting the data. The MVIC value of the subjects was measured after attaching the EMG, and calibration was performed after attaching the MyoMotion. The EMG value was measured for 10 sec during rest respiration with the subject in both the correct sitting posture and the crossed leg posture. The peak EMG value was presented as a percentage of MVIC after attaching the EMG pad to the muscle and this is shown as the collected data. Using EMG and MyoMotion, the tape was used to measure the mobility of the chest wall at the time of rest respiration and at the time of maximum inspiration in the correct sitting posture. Also, the FVC and FEV1 were measured using a spirometer. The measurement was performed with the subject in the correct sitting position, followed by 30 minutes of rest [21]. Chest wall mobility and pulmonary function were measured using the same method as that used with the subject in the crossed leg position. The order of correct sitting posture and the crossed leg sitting posture was randomly assigned.

\section{Statistic analysis}

All the data was analysed using SPSS version 22.0 (SPSS Inc., USA). The data was expressed as mean \pm standard deviation (SD). A paired t-test was used to compare the correct sitting posture with the cross-legged sitting postures. The Kolmogorov-Smirnov (K-S) test was used to compare the distributions of variables with standard normal distributions. All the data was normally distributed. The statistical significance level was set at $\mathrm{p}<.05$.

\section{Results}

\section{Motion analysis}

Table 1 shows a comparison of the vertebral angle according to the sitting posture. The thoracic flexion was statistically significantly decreased, and the lumbar flexion was statistically significantly increased according to the subjects' sitting posture $(\mathrm{p}<.05)$.

\section{Chest wall mobility}

Table 2 shows the results of the chest wall mobility with the subjects in the correct sitting posture and also in the crossed leg posture. During rest respiration, the value in the correct sitting posture was $95.52 \pm 1.09$, and the value in the crossed leg posture was $94.55 \pm 9.86(\mathrm{p}<.05)$. During 
Table 1. Comparison of Vertebrae Angle According to Sitting Posture

\begin{tabular}{cccc}
\hline & Correct Sitting Posture $(\mathrm{deg})$ & Crossed Leg Posture $(\mathrm{deg})$ & $p$ \\
\hline Thoracic Flexion & $-6.90 \pm 7.25^{\mathrm{a}}$ & $-12.54 \pm 7.96$ & $.000^{*}$ \\
Thoracic Lateral & $-1.05 \pm 3.81$ & $-.77 \pm 3.63$ & .580 \\
Thoracic Axial & $-.86 \pm 10.53$ & $-.92 \pm 5.21$ & .977 \\
Lumbar Flexion & $20.95 \pm 13.31$ & $32.08 \pm 13.89$ & $.001^{*}$ \\
Lumbar Lateral & $2.66 \pm 3.79$ & $2.50 \pm 3.68$ & .842 \\
Lumbar Axial & $1.79 \pm 9.90$ & $-.95 \pm 3.13$ & .154 \\
\hline
\end{tabular}

${ }^{\mathrm{a}} \mathrm{Mean} \pm \mathrm{SD} * \mathrm{p}<.05,(+)$ value $=$ flexion, (-)value $=$ extension

Table 2. Comparison of Chest Wall Mobility According to the Sitting Posture

\begin{tabular}{lcccc}
\hline & Correct Sitting Posture & Crossed Leg Posture & $t$ & $p$ \\
\hline Rest Respiration $(\mathrm{cm})$ & $94.55 \pm 9.86^{\mathrm{a}}$ & $95.52 \pm 10.09$ & -3.616 & $.001^{*}$ \\
Peak Inspiration $(\mathrm{cm})$ & $97.24 \pm 9.63$ & $98.00 \pm 9.77$ & -3.884 & $.001^{*}$ \\
\hline
\end{tabular}

${ }^{\mathrm{a}} \mathrm{Mean} \pm \mathrm{SD} * \mathrm{p}<.05$

Table 3. Comparison of Pulmonary Function According to the Sitting Posture

\begin{tabular}{ccccc}
\hline Variables & Positioning & Mean \pm SD & $t$ & $p$ \\
\hline \multirow{2}{*}{ FVC (L) } & Correct Sitting Posture & $3.99 \pm .94$ & .029 & .977 \\
& Crossed Leg Posture & $3.99 \pm .96$ & & $.2 .83 \pm .86$ \\
\multirow{2}{*}{ FEV1(L) } & Correct Sitting Posture & $2.70 \pm .85$ & 1.346 & .188 \\
& Crossed Leg Posture & & \\
\hline
\end{tabular}

FVC: Forced Vital Capacity, FEV1: Forced Expiratory Volume

peak inspiration, the value in the correct sitting posture was $98.00 \pm 9.77$, and the value in the crossed leg posture was $97.24 \pm 9.63(\mathrm{p}<.05)$. These two values were significantly decreased.

\section{Pulmonary function}

Table 3 shows the results for the pulmonary function with the subjects in the correct sitting posture and also in the crossed leg posture. There was no significant difference between the FVC and FEV1.

\section{Muscles activity}

Table 4 shows the results of respiratory muscles' activity with the subjects in the correct sitting posture and also in the crossed leg posture. The right EO was $1.34 \pm .89$ with the subjects in the correct sitting posture and $.99 \pm .76$ with the subjects in the crossed leg posture $(p<.05)$. The left IO was $1.58 \pm 1.49$ with the subjects in the correct sitting posture and $.99 \pm .80$ with the subjects in the crossed leg posture $(\mathrm{p}<.05)$. There was also a significant decrease in the right EO and left IO values when comparing the correct and crossed leg sitting postures $(\mathrm{p}<.05)$.

\section{Discussion}

The angle of thoracic flexion decreases significantly and 
Table 4. Comparison of the Respiratory Muscles' Activity According to the Sitting Posture

\begin{tabular}{cccccc}
\hline & & Correct Sitting Posture & Crossed Leg Posture & $t$ & $p$ \\
\hline SCM & Right & $.81 \pm .52^{\mathrm{a}}$ & $.78 \pm .46$ & .490 & .627 \\
$(\% \mathrm{MVICb})$ & Left & $.78 \pm .63$ & $.87 \pm .78$ &. .834 & .411 \\
\hline RA & Right & $.95 \pm .96$ & $.77 \pm .57$ & 1.198 & .240 \\
$(\% \mathrm{MVIC})$ & Left & $.91 \pm .95$ & $.81 \pm .89$ & 1.401 & .171 \\
\hline EO & Right & $1.34 \pm .89$ & $.99 \pm .76$ & 2.811 & $.009^{*}$ \\
$(\% \mathrm{MVIC})$ & Left & $1.43 \pm .88$ & $1.25 \pm 1.00$ & 1.729 & .094 \\
\hline IO & Right & $1.26 \pm .94$ & $.89 \pm .90$ & 2.367 & $.025^{*}$ \\
$(\% \mathrm{MVIC})$ & Left & $1.58 \pm 1.49$ & $.99 \pm .80$ & 2.257 & $.015^{*}$ \\
\hline
\end{tabular}

${ }^{\mathrm{a}} \mathrm{Mean} \pm \mathrm{SD}{ }^{*} \mathrm{p}<.05,{ }^{\mathrm{b}}$ maximal voluntary isometric contraction, $\mathrm{SCM}$ : sternocleidomastoid, RA: rectus abdominis, EO: external oblique, IO: internal oblique

the lumbar flexion increases significantly in the crossed leg posture. Further, the chest wall mobility significantly decreases in the crossed leg posture. There was no significant difference in the pulmonary function, but the muscle activity of the right EO and both IOs significantly decreased.

This study showed that the thoracic flexion angle was significantly extended and the lumbar flexion angle was more flexed in the crossed leg posture. Similar to that seen in our study, the crossed leg posture was previously found to cause lumbar flexion in the study by [22], and lumbar spine flexion was significantly greater in the crossed leg posture than that previously noted in the correct sitting posture [23]. However, according to previous studies, thoracic flexion was significantly increased, and there was kyphosis in the thorax when the subjects sat in the crossed leg posture $(\mathrm{p}<.05)$ [22]. A possible explanation for the different thoracic vertebral angles when comparing our results to those of previous studies is the difference in the measurement procedure. In our study, we measured the thoracic angle immediately with 3D motion analysis when the subject crossed his/her legs. Thus, the crossed leg posture caused lumbar flexion, yet thoracic flexion could present differently depending upon the time when the measurement was taken or the conditions that were present.

The results of our study show that chest wall mobility at rest and during peak inspiration was significantly decreased with the subjects sitting in the crossed-leg posture as compared to that of the subjects in correct sitting posture. Although there have been no studies investigating chest wall mobility in the crossed-leg posture, the altered lumbar and thoracic angles would affect the mobility of the chest wall. A previous study claimed that the pelvis tilts to the lateral side in the crossed-leg posture, and the chest compensation mechanism on the lateral side is visible [2]. Pelvic asymmetry causes musculoskeletal problems by changing the body's mechanics and increasing the deformation of soft tissues and bones [24]. Also, the vertebral deformity is at the limit of vertebral rotation, the limit of thoracic deformity, and the limits of the respiratory muscles, and so all of this distorts the dynamics of the chest and impairs various body functions [25]. Therefore, when the lumbar and thoracic vertebrae are not aligned, there will be a reduction of chest wall mobility. Also, chest wall deformity impairs the respiratory function because it affects lung growth [26]. Although this study shows no significant difference between the FVC and 
FEV1, some studies claim that sitting with a poor posture will have a detrimental effect on the musculoskeletal system [27]. Further, several studies have found associations between spinal alignment and changes in pulmonary function [28]. The reason for the lack of a significant difference between the FVC and FEV1 may have been that this study recruited only healthy adults. If patients suffering with respiratory problems had been assessed, the results would have been quite different.

This study also showed a significant decreased in the right EO and IO values and the left $\mathrm{IO}$ and $\mathrm{EO}$ values $(\mathrm{p}<.05)$. Likewise, the right EO and IO muscle activities were significantly lower in the crossed leg posture than that in the correct sitting posture $(\mathrm{p}<.05)$, and the left IO muscle activity was also significantly decreased in the crossed leg posture $(p<.05)$ [29]. It has been noted that the external oblique abdominal muscle is less active during sitting with the legs crossed [30]. The advantages of the crossed leg posture are reduced fatigue when working in a sitting position for a long time by reducing the muscular activity and increased stability in the ceiling joint [30]. However, a crossed leg posture sustained for a long time may cause muscle imbalance, so sitting with a crossed leg posture should be avoided as this can aggravate muscle imbalance.

There are some limitations to the interpretation of our results. First, it is difficult to generalize the results of this study to all subjects because the findings here are specific to healthy adults and not patients. Second, although the participants were verbally instructed to minimize movement in order to reduce the noise of the electrical signal, the influence of movement during postural change could not be completely excluded. Third, this study was conducted over a short period of time. Thus, in the future, it may be necessary to conduct a study on those patients with impaired respiratory function.

\section{Conclusion}

The crossed leg posture caused slight thoracic extension and lumbar flexion, and this alteration may reduce chest wall mobility. The crossed leg posture also causes abdominal muscle imbalance, and so sitting with a crossed leg posture should be avoided as it can alter the sitting alignment in the vertebral spine, reduce the mobility of the chest wall, and aggravate abdominal muscle imbalance. However, changes in the vertebral spine angle, reduced chest wall mobility, and activation of imbalance of the EO and IO due to a crossed leg sitting posture did not have any clinical effect on the pulmonary function of healthy people. The results of this study show that short periods of assuming a crossed leg sitting posture does not affect the pulmonary function of healthy adults. In the future, it may be necessary to study the effects over an extended period of time of sitting with crossed legs sitting for patients with impaired respiratory function.

\section{References}

[1] Park YN, Bae YS. Comparison of postures according to sitting time with the leg crossed. J Phys Ther Sci. 2014;26(11):1749-52.

[2] Ahn SJ, Kim SH, Kang SY, et al. Asymmetrical change in the pelvis and the spine during cross-legged sitting postures. J Korean Med Sci. 2013;27(11):3427--2.

[3] Gimson M. Weight distribution in sitting and the effect of leg-crossing. J Physiother. 2003;89(2):125.

[4] Woo HS, Oh JC, Won SY. Effects of asymmetric sitting on spinal balance. J Phys Ther Sci. 2016;28(2):355-9.

[5] Andersson BJ, Ortengren R, Nachemson AL, et al. The sitting posture: an electromyographic and discometric study. Orthop Clin North Am. 1975;6(1):105-20.

[6] Kim MJ, Son CG, Heo DS, et al. Analysis of clinical tendency of spinal disorder in primary, middle and high school students in Korea. The Acupuncture. 2010;27. 
[7] Young CIJ, Crocker MJ. Acoustical analysis, testing, and design of flow-reversing muffler chambers. J Acoust Soc Am. 1976;60(5):1111-8.

[8] Jubran A, Tobin MJ. The Effect of Hyperinflation on Rib Cage-Abdominal Motion1-3. Am Rev Respir Dis. 1992;146:1378-82.

[9] Bryant EC, Saber-Sheikh K, Glazzard C, et al. Feasibility of using inertial sensors to assess human movement. J Man Manip Ther. 2010;15(1):122-5.

[10] Struzik A, Konieczny G, Stawarz M, et al. Relationship between lower limb angular kinematic variables and the effectiveness of sprinting during the acceleration phase. Appl Bionics Biomech. 2016.

[11] Xie YF, Szeto G, Madeleine P, et al. Spinal kinematics during smartphone texting-A comparison between young adults with and without chronic neck-shoulder pain. Appl Ergon. 2018;68:160-8.

[12] Bockenhauer SE, Chen H, Julliard KN, et al. Measuring thoracic excursion: reliability of the cloth tape measure technique. J Am Osteopath Assoc. 2007;107(5):191-6.

[13] Lanza FdC, de Camargo AA, Archija LRF, et al. Chest Wall Mobility Is Related to Respiratory Muscle Strength and Lung Volumes in Healthy Subjects. AARC. 2013; 58(12):2107-12.

[14] Pradnya D, Nalini A, Adoor M, et al. Respiratory Dysfunctions in Children with Duchenne muscular dystrophy. Int J Physiother Res. 2016;4(1):1365-69.

[15] Ranu H, Wilde M, Madden B. Pulmonary Function Tests. Ulster Med J. 2011;80(2):84-90.

[16] Han J, Park S, Kim Y, et al. Effects of forward head posture on forced vital capacity and respiratory muscles activity. J Phys Ther Sci. 2016;28(1):128-31.

[17] Criswell E. Cram's introduction to surface electromyography. Jones and Bartlett Publishers. 2010.

[18] Cram JR. Introduction to surface electromyography. Aspen Publishers. 1998.

[19] Choi WJ, Robinovitch SN, Ross SA, et al. Effect of neck flexor muscle activation on impact velocity of the head during backward falls in young adults. Clin Biomech. 2017;49:28-33.

[20] Escamilla RF, McTaggart MS, Fricklas EJ, et al. An electromyographic analysis of commercial and common abdominal exercises: implications for rehabilitation and training. J Orthop Sports Phys Ther. 2006;36(2):45-57.

[21] Lee Bk. The Effect of the Forced Pulmonary Function of young female, by Changes in Lung function Related to Postures and by Transverse Abdominis Activation in Standing Position. JKSPM. 2012;7(3):267-74.

[22] Tully EA, Fotoohabadi MR, Galea MP. Sagittal spine and lower limb movement during sit-to-stand in healthy young subjects. Gait posture. 2005;22(4):338-45.

[23] Yu JS, An DH. Differences in lumbar and pelvic angles and gluteal pressure in different sitting postures. J Phys Ther Sci. 2015;27(5):1333-35.

[24] Egan DA, Al-Eisa E. Pelvic skeletal asymmetry, postural control, and the association with low back pain: a review of the evidence. Crit Rev Phys Rehabil Med. 1999;11(3,4).

[25] Cooper DM, Rojas JV, Mellins RB, et al. Respiratory mechanics in adolescents with idiopathic scoliosis. Am Rev Respir Dis. 1984;130(1):16-22.

[26] Campbell R, Smith MD. Thoracic insufficiency syndrome and exotic scoliosis. J Bone Joint Surg Am. 2007;89(Suppl 1):108-22.

[27] Pynt J, Higgs J, Mackey M. Seeking the optimal posture of the seated lumbar spine. Physiother Theory Pract. 2001;17(1):5-21.

[28] Nwaobi OM, Smith PD. Effect of adaptive seating on pulmonary function of children with cerebral palsy. Dev Med Child Neurol. 1986;28(3):351-4.

[29] Jung YG. Effects of leg crossed sitting on the trunk muscle activities during the computer work. Yonsei University ( $\mathrm{PhD}$ Thesis). Dissertation of master's degree. 2005.

[30] Snijders CJ, Slagter AH, Vleeming A, et al. Why leg crossing? The influence of common postures on abdominal muscle activity. Spine. 1995;20(18):1989-93. 